\title{
Design of a convolutional neural network for classification of biomedical signals
}

\section{Diseño de una red neuronal convolucional para la clasificación de señales biomédicas}

\author{
JALOMO, Jaime $\dagger^{*}$, PRECIADO, Edith and GUDIÑO Jorge \\ Tecnologico Nacional de México - Instituto Tecnologico de Cd. Guzmán, Departamento de Maestría en Ingeniería \\ Electrónica; Maestría en Ingeniería Electrónica y Universidad de Colima, Facultad de Ingeniería Electromecánica.
}

ID $1^{\text {st }}$ Author: Jaime, Jalomo / ORC ID: 0000-0003-0246-299X, arXiv Author ID: JaimeJalomo, PubMed Author ID: JaimeJalomo, CVU CONACYT ID 36269

ID $1^{\text {st }}$ Coauthor: Edith, Preciado / ORC ID 0000-0002-6333-5583, arXiv Author ID EdithPreciado, PubMed Author ID EdithPreciado, CVU CONACYT ID 905025

ID $2^{\text {nd }}$ Coauthor: Jorge, Gudiño / ORC ID 0000-0002-0585-908X, arXiv ID: jorgeglau, Researcher ID Thomson: Q-68442018, PubMed ID: jorgeglau y CVU CONACYT ID: 122644

DOI: $10.35429 / J R D .2020 .17 .6 .15 .20$

Received: January 21, 2020; Accepted: June 24, 2020

\begin{abstract}
Biomedical signals are current case of Avant-garde study, thanks to advances in artificial intelligence, every day new methods are implemented that are useful for the treatment of this signals, mainly to detect anomalies or diseases with greater precision. A solution on base of the Deep Learning is proposed, this technology has proven to be efficient in handling high-level feature data, in it featured neural networks convolutionals (NNC) which are ideal in image management. In this paper, electrocardiographic signals (ECG) designed from a dynamic mathematical model in a two convolution layer NNC for classification are used.
\end{abstract}

Neural Network Convolutional (NNC), ECG, Dinamical model, Classification

\begin{abstract}
Resumen
Las señales biomédicas actualmente son un caso de estudio vanguardista, gracias a los avances en temas de inteligencia artificial, día a día se implementan nuevos métodos que sean de utilidad para el tratamiento de estas señales, principalmente para detectar anomalías o enfermedades con mayor precisión. Se propone la solucion de la tecnología deep Learning (Aprendizaje profundo) la cual ha demostrado ser eficiente en el manejo de datos de características de alto nivel, en ella destacan las Redes Neuronales Convolucionales (RNC) las cuales son ideales en el manejo de imágenes. En el presente trabajo se emplean señales electrocardiográficas (ECG) diseñadas a partir de un modelo dinámico matemático en una RNC de cuatro capas de convolución para su clasificación.
\end{abstract}

Red Neuronal Convolucional (RNC), ECG, Modelo Dinámico, Clasificación

Citation: JALOMO, Jaime, PRECIADO, Edith and GUDIÑO Jorge. Design of a convolutional neural network for classification of biomedical signals. Journal of Research and Development. 2020. 6-17: 15-20.

\footnotetext{
* Correspondence to the Author (Email: jaimejc@itcg.edu.mx)

$\dagger$ Researcher contributing as first author.
} 


\section{Introduction}

The analysis of biomedical signals has generally been carried out by the own inspection of health professionals, it is their word and criteria that determine the state of these signals. Today, we are working at an accelerated pace in the development of algorithms that are helpful in carrying out this work, thus providing a reliable support tool for the diagnosis of these signals. The data of the biomedical signals with which these algorithms are fed are mostly obtained from some database, acquiring them on your own is usually a challenge if you do not have the necessary equipment or authorization by means of an "informed consent" document from Test subjects to sample the signals, for the most part, the data obtained from medical equipment are usually encoded and it results in extra work having to decode them. A reliable way to acquire these signals and thus be able to implement them in the development of these algorithms is through the implementation of some dynamic model.

Currently, thanks to advances in Convolutionary Neural Networks, they have been at the forefront of pattern recognition applications, they can even exceed human capacity, as Graham shows in his article Fractional Max-Pooling [1]. This neural network is ideal for working with images, which play a very important role in computational vision. The image classification process includes image preprocessing, image segmentation, extraction of key characteristics and identification of similarities.

This article proposes the classification of biomedical images, in this case the ECG electrocardiographic signal using a convolutional neural network, using the mathematical dynamic model of the ECG signal proposed by Patrick E. McSharry [2] for the design and generation of the images.

In the next two sections of this document, the topics of "Convolutionary Neural Networks" and "Biomedical Signal ECG Using a Dynamic Model" are covered, the main topics required for the development of this work, in a later section the methodology for the development of the work (ECG biomedical signal classification by Convolutionary Neural Network) to finally detail the results obtained and conclusions in the last two sections.

\section{Convolutional Neural Networks}

Convolutional neural networks (RNCs) are very useful in practical applications, since 2012 these networks have become the algorithm for all computational vision tasks, generally working with all perceptual tasks. [3] They specialize in processing data that has a known grid topology, "time-series data" is considered to be a 1D grid that samples at regular time intervals, and "image data" is considered a 2D pixel grid . These types of networks are based on a mathematical operation called convolution because they perform the convolution of the input signal with respect to a transfer function that is found at the output of the neuron.

Their attributes make them perfect for analyzing images, one of the problems that any Deep Learning architecture suffers mainly is the large number of weights since they compare the pattern learned with respect to its input, assuming that the input is an image (Height $\mathrm{x}$ Width $x$ Channels) each neuron would learn as many weights as pixels the image has, which is technically difficult.

The images replicate their most basic patterns quite a bit, which makes the convolutional neural network easier since it (2D convolutional for images) will basically look for the same pattern, but in different positions of the image.

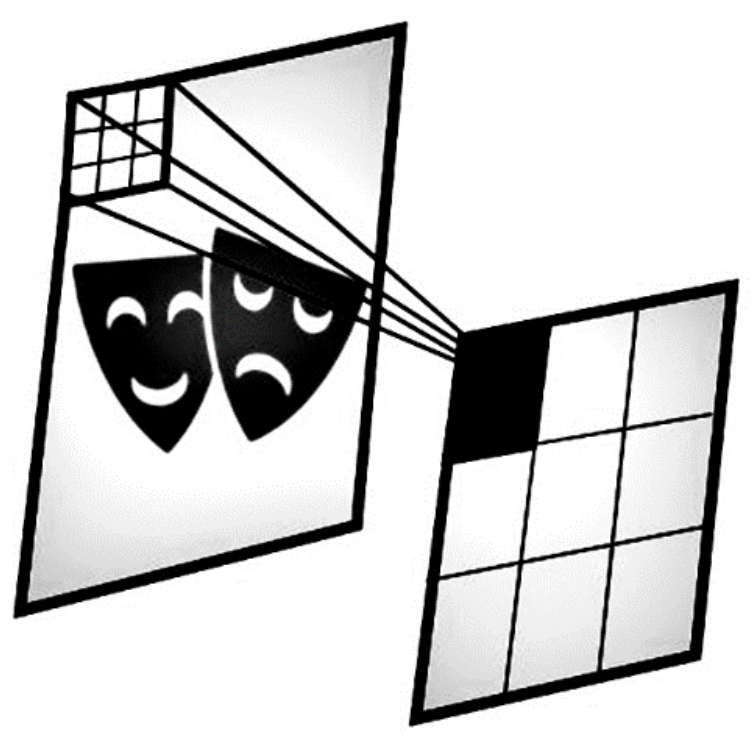

Figure 1 Looking for a pattern of an image 
There are three types of layers that make up a convolutional neural network: convolutional layer, pooling layer and fullyconnected layer.

\section{Convolutionary Layer}

It is the most important layer of an RNC, the neurons of the first convolutional layer are not connected to each of the pixels of the input image, but only to those that are within its receptive field. In the second convolutional layer each neuron is connected only to the neurons located in a small rectangle in the first layer. What sets this layer apart from the FullyConnected layer is that the latter does have all of its neurons connected to the neurons in the previous layer.

This architecture allows the network to focus on low-level features in the first hidden layer, then assemble them into higher-level features in the next hidden layer, and so on.

\section{Pooling layer}

The objective of this layer is to subsample the input image in order to reduce the number of neural network parameters to be used, which benefits in reducing the computational load and the memory use of the equipment.

As in convolutional layers, in the pooling layer each neuron is connected to the output of a limited number of neurons in the previous layers, located within a small rectangular receptive field. This layer has no weights, all it does is get the maximum value (max-pooling) or the average value of all that window (avg-pooling).

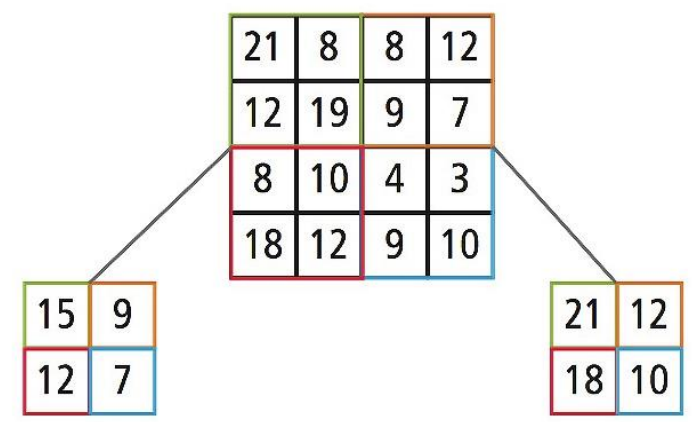

\section{Average Pooling}

Max Pooling

Figure 2 Pooling layer

Source: (https://stats385.github.io/poolinglayers)

\section{Biomedical ECG signal using Dynamic Model}

The ECG electrocardiographic signal is variable in time, it reflects the flow of ionic current that causes the heart fibers to contract and subsequently relax. This signal is obtained by recording the potential difference between two electrodes placed on the skin of the test subject or patient.

At the present time, the development and implementation of different algorithms for treating biomedical signals is continuously advancing, the dynamic model for the generation of synthetic electrocardiographic signals facilitates the evaluation of these algorithms without the need to resort to a data bank or generate the signs.

\section{Dynamic Model}

The model generates a trajectory in a threedimensional space (3-D) with coordinates ( $x, y$, $\mathrm{z}$ ), with realistic PQRST morphology and described dynamic heart pulse. The purpose of this model is to provide a realistic standard ECG signal with known characteristics, which facilitates the comparison of different signal processing techniques.

Dynamic equations of motion are given by a set of three ordinary differential equations: [4]

$\dot{x}=\propto x-\omega y$

$\dot{y}=\propto y-\omega x$

$\dot{z}=-\sum_{i \in\{P, Q, R, S, T\}} a_{i} \Delta \theta_{i} \exp \left(-\frac{\Delta \theta_{i}^{2}}{2 b_{i}^{2}}\right)-\left(z-z_{0}\right)$

For the study of the parameters of this model, the revision of [4] is suggested since its description is significantly extensive. Both $\dot{x}, \dot{y}$ and $\dot{z}$ are discussed in detail in the reference [4].

\section{Biomedical ECG signal classification using} the Convolutional Neural Network

\section{ECG signal}

For the generation of the signals that were used in the training of our RNC, the dynamic model of the ECG signal was programmed using embedded Simulink blocks. 

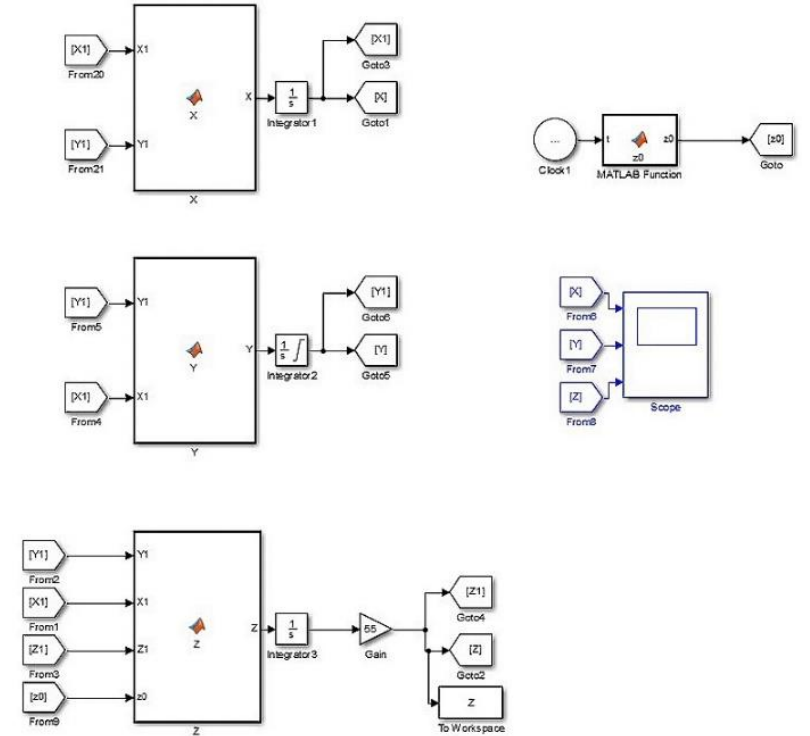

Figure 3 Modelo dinámico señal ECG programado en simulink

The following images show an ideal PQRST wave generated by the dynamic model, with the characteristic that its $\mathrm{x}$ and $\mathrm{y}$ axes are unitary.

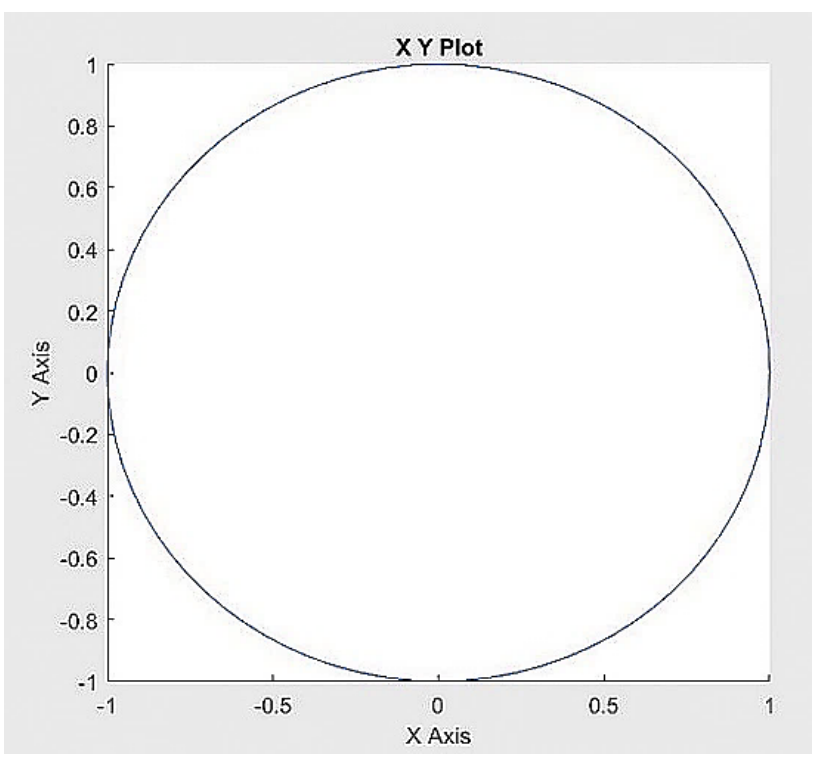

Figure 4 Unit circle generated by equations $\mathrm{x}$ and $\mathrm{y}$

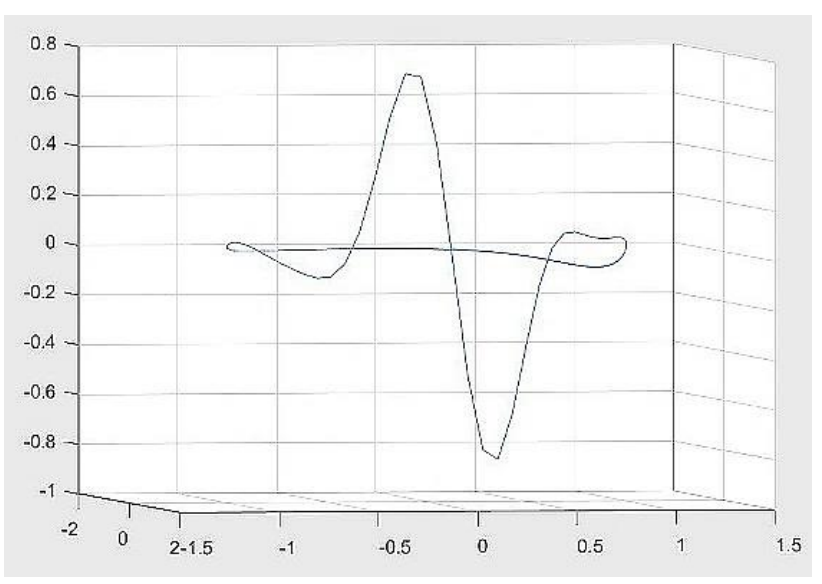

Figure 5 Typical path generated by the dynamic model (1) in the given 3-D space $(x, y, z)$
$\underline{R N C}$

The classification to be made is of a binary type, that is, the RNC will show us if the ECG image that is being analyzed is of the abnormal type = 0 or normal $=1$. The sequential model is used, which refers to the creation of a series of layers of sequential neurons (one after the other). Images are resized to $300 \times 150$ pixels. In the present work, the Google Collaboratory tool was used to make the results of our training more efficient, in this case decrease the loss and increase its accuracy, as mentioned in the article "Google Collaboratory as an alternative for the processing of a convolutional neural network "[8], in addition to being able to make use of computer tools with better characteristics such as a GPU which are not found in all conventional and standard equipment.

\section{A. RNC architecture flow chart}

The characteristics of the neural network architecture consist of 2 blocks with 2 convolutional layers and 1 MaxPooling layer each as shown in Figure below

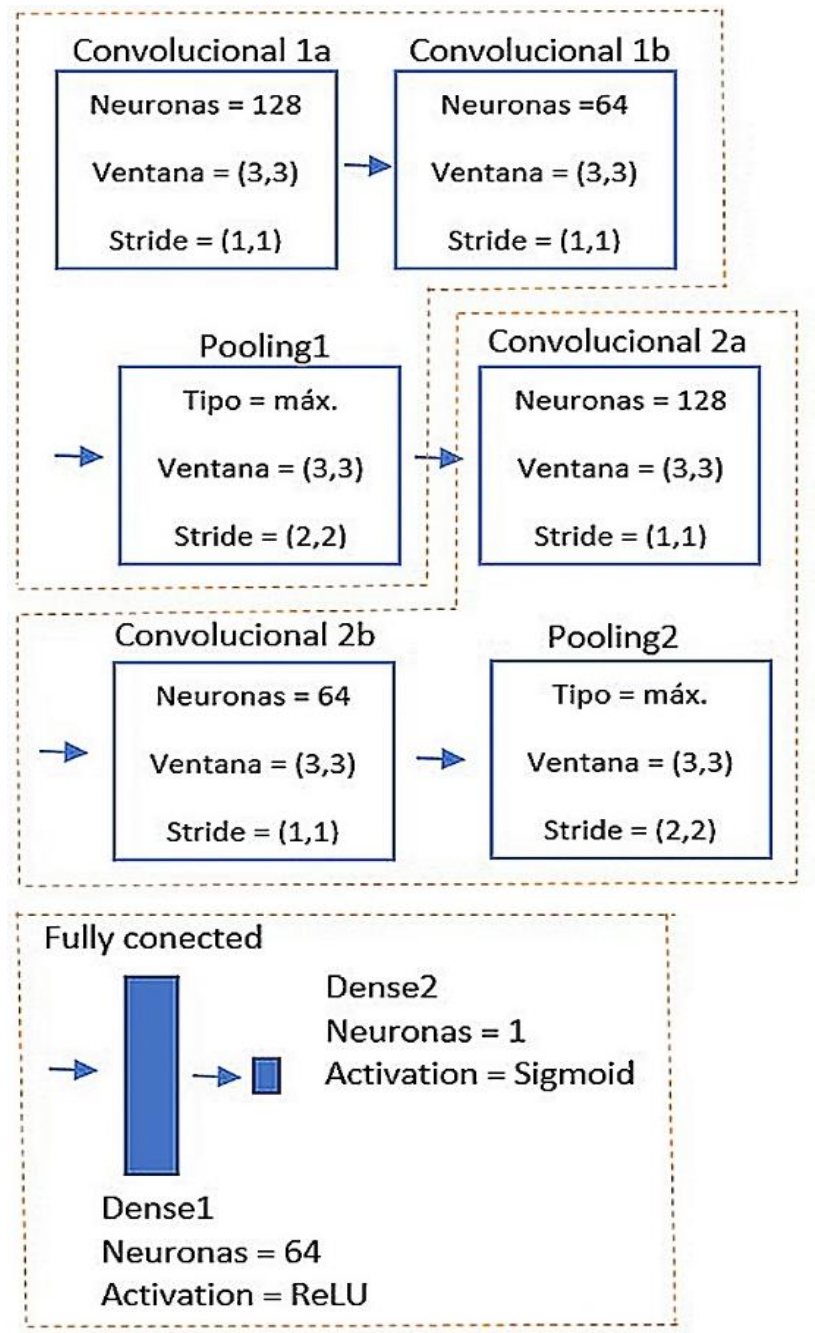

Figure 6 RNC architecture

JALOMO, Jaime, PRECIADO, Edith and GUDIÑO Jorge. Design of a convolutional neural network for classification of biomedical signals. Journal of Research and Development. 2020 
The convolutional layers consist of 128 neurons and 64 neurons respectively, as well as a window $(3,3)$ and a stride $(1,1)$, for MaxPooling layers the stride is used as $(2,2)$. Subsequently a third block consisting of 2 Fully connected layers, in the last layer only a sigmoid neuron will be used to represent the probability of being normal or abnormal.

The network trains for 30 epochs where each one will have 1000 iterations. The iterations increase each time the weights are updated and the times increase each time all the samples of the dataset have been learned and it starts again, this means that they will be passed 30 times through the same example. The signals generated to train, validate and test our RNC were filed in 3 different folders, TRAINING, VALIDATION and TEST.

\section{Results}

The results of the training of the network were favorable since the precision (accuracy) which indicates the performance that our model is having was increasing until reaching the values of 0.99 , while the loss was decreasing until reaching the value of 0.00023 , the latter indicates the difference between the value predicted by the model and the true value, which can be seen in the following graphs.

\section{LOST}

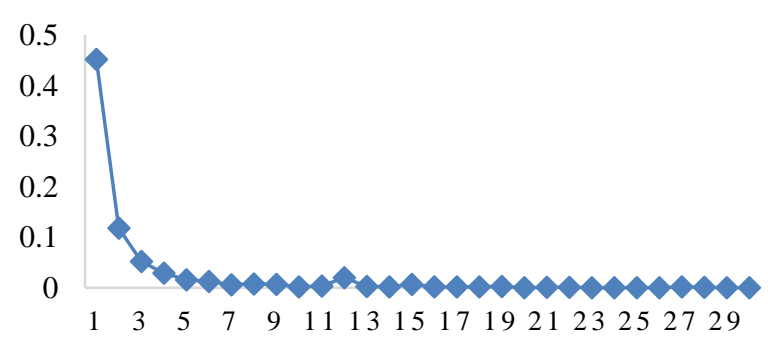

Graphic 1 Lost

\section{PRECISION}

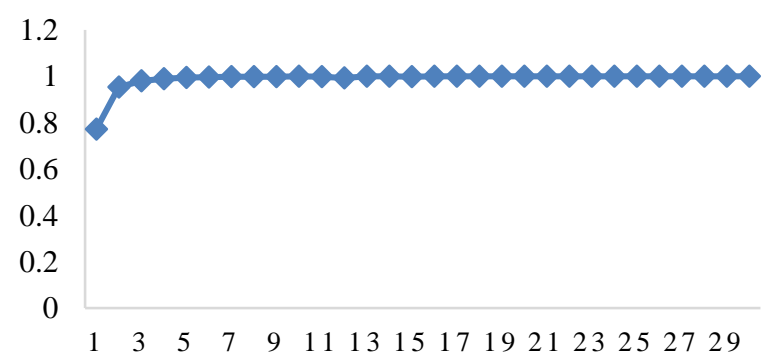

The network was tested with 27 images of normal and abnormal signals which were classified correctly in their entirety, below are some of the images which were classified correctly, 3 of abnormal type and 3 of normal type, it should be noted that these images do not belong to the data set with which the convolutional neural network was trained.

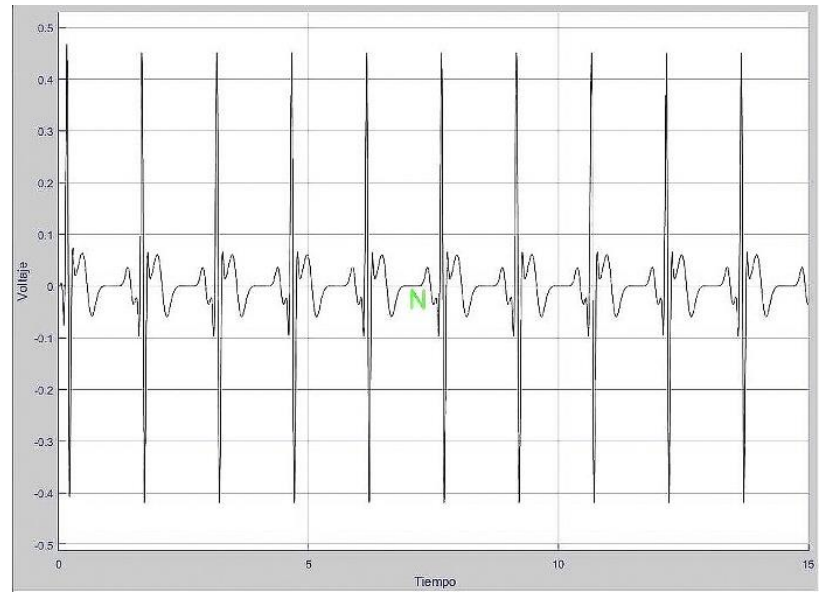

Figure 7 Result "Normal" 1

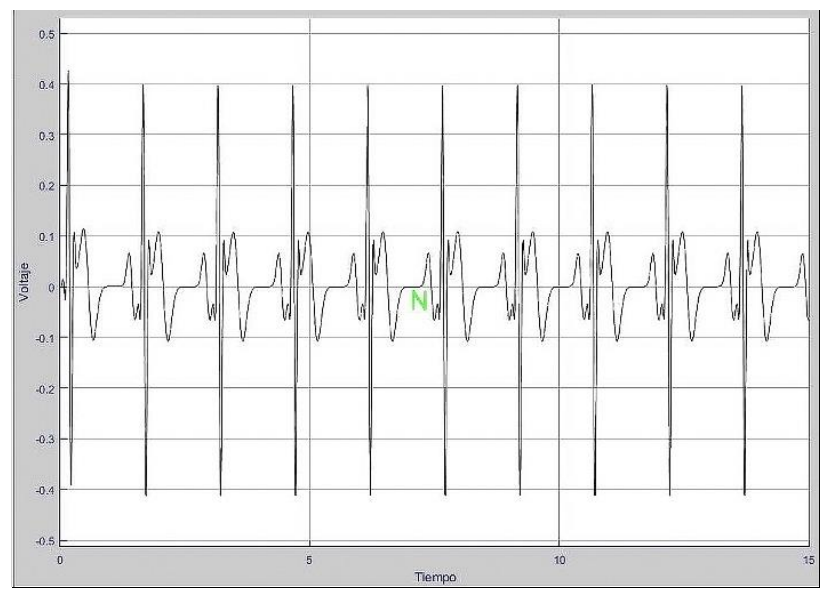

Figure 8 Result "Normal" 2

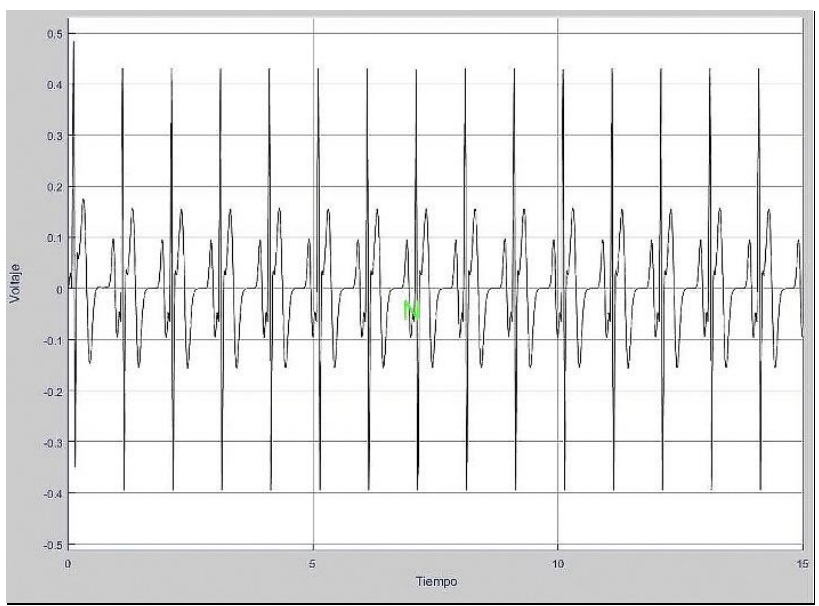

Figure 9 Result "Normal" 3 


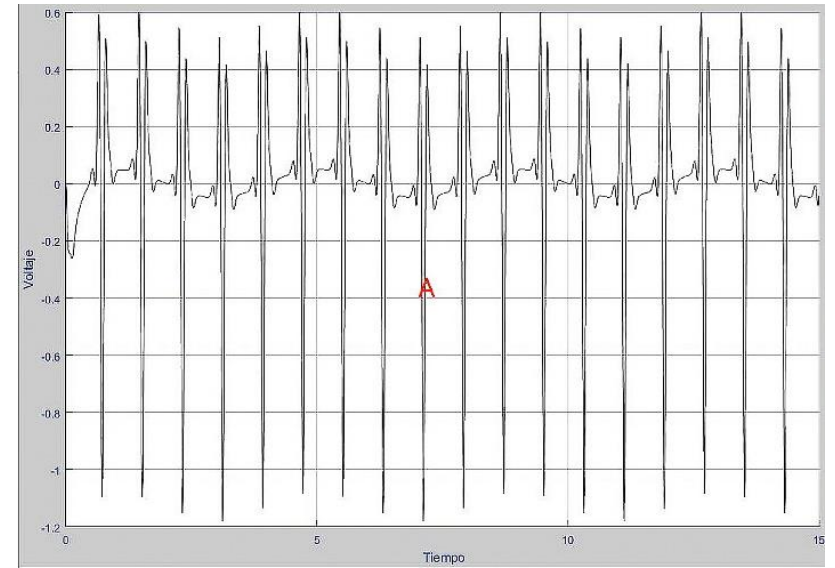

Figure 10 Result "Abnormal" 1

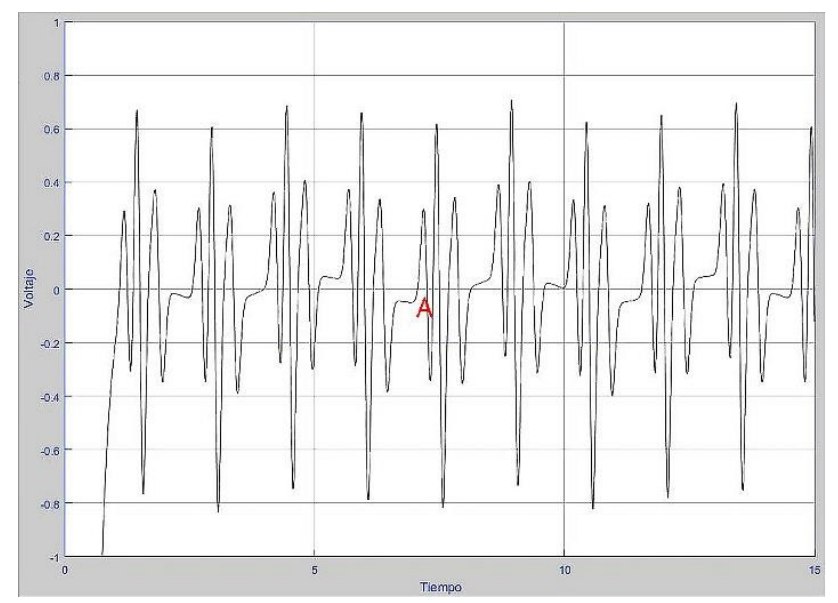

Figure 11 Result "Abnormal" 2

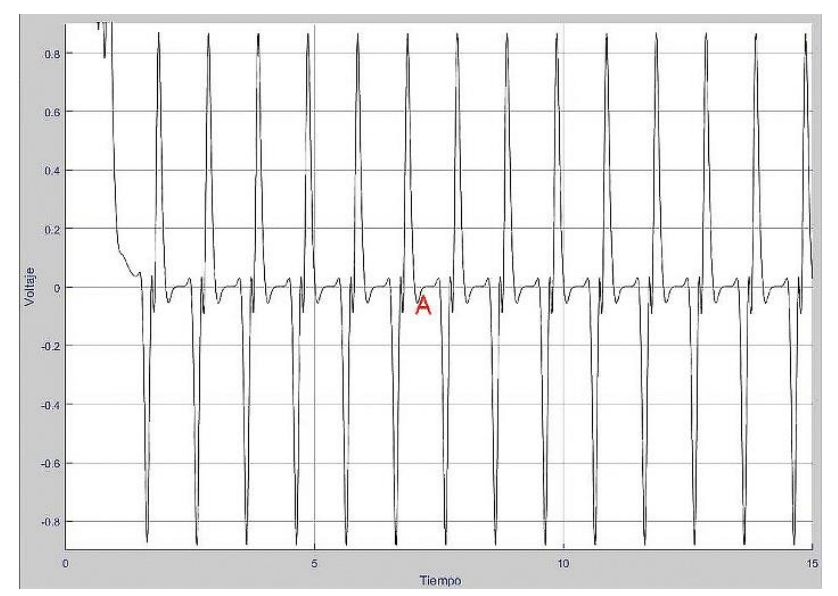

Figure 12 Result "Abnormal" 3

\section{Conclusions}

The results obtained allow us to corroborate how the technology of the Convolutionary Neural Networks is capable of analyzing various types of data equal to or more efficiently than human capacity, in this case classifying the images of the ECG signal as a professional would do. from health to visual inspection.
The training used for the network was sufficient for the classification performed, in this first case it was a simple classification with clear ECG images and without any noise, as real signal images would have, for this case study with noise it is necessary to modify these parameters network training, as well as its architecture parameters. The classification between ECG signal images by binary method is sufficient to be able to differentiate whether a signal is at rest or effort, as well as healthy or with a health problem. Aspects to consider and in which work has already begun is the management of real signals, as well as applying it to a medical case (which may be arrhythmia, cardiomyopathy, among others).

\section{References}

[1] B. Graham, (2015). "Fractional MaxPooling", arXiv:1412.6071.

[2] E. Mcharry, Gari D, “A Dynamical Model for Generating Synthetic Electrocardiogram Signals", IEEE Trans.Biomed. Eng, vol. 50. March 2003.

[3] F. Chollet, DEEPLEARNING with Python. MANNING Publications Co, Shelter Island, NY 2018.

[4] E. Mcharry, Gari D, “A Dynamical Model for Generating Synthetic Electrocardiogram Signals", IEEE Trans.Biomed. Eng, vol. 50. March 2003, pp 291.

[5] A. Géron, Hands-On Machine Learning with Scikit-Learn \& TensorFlow. O'REILLY, Shelter Island, United States of América 2017.

[6] I. Goodfellow, Y. Bengio and A. Courville, Deep Learning. MIT Press, 2016. http://www.deeplearningbook.org.

[7] T. Guo, J. Dong, H. Li, Y. Gao, "Simple Convolutional Neural Network on Image Classification", IEEE Int. Conf. Big Data Analysis. Eng. March 2017. doi: 10.1109/ICBDA.2017.8078730

[8] BODERO, E. M., LOPEZ, M. P., CONGACHA, A. E., CAJAMARCA, E. E., \& MORALES, C. H. "Google Colaboratory como alternativa para el procesamiento de una red neuronal convolucional". Revista ESPACIOS, 2020.

JALOMO, Jaime, PRECIADO, Edith and GUDIÑO Jorge. Design of a convolutional neural network for classification of biomedical signals. Journal of Research and Development. 2020 